\title{
State-of-Charge Estimation of Lithium-Ion Battery Pack Based on Improved RBF Neural Networks
}

\author{
Li Zhang $\mathbb{D}^{1}{ }^{1}$ Min Zheng $\left(\mathbb{D},{ }^{1}\right.$ Dajun Du $\mathbb{D}^{1},{ }^{1}$ Yihuan Li, $^{2}$ Minrui Fei, ${ }^{1}$ Yuanjun Guo, \\ and Kang $\mathrm{Li}^{2}$ \\ ${ }^{1}$ Shanghai Key Laboratory of Power Station Automation Technology, School of Mechatronic Engineering and Automation, \\ Shanghai University, Shanghai 200072, China \\ ${ }^{2}$ University of Leeds, Leeds LS2 9JT, UK \\ ${ }^{3}$ Shenzhen Institute of Advanced Technology, Chinese Academy of Sciences, Shenzhen 518055, China
}

Correspondence should be addressed to Min Zheng; zhengmin203@163.com

Received 9 August 2020; Revised 22 September 2020; Accepted 24 October 2020; Published 1 December 2020

Academic Editor: Jing $\mathrm{Na}$

Copyright (C) 2020 Li Zhang et al. This is an open access article distributed under the Creative Commons Attribution License, which permits unrestricted use, distribution, and reproduction in any medium, provided the original work is properly cited.

\begin{abstract}
Lithium-ion batteries have been widely used as energy storage systems and in electric vehicles due to their desirable balance of both energy and power densities as well as continual falling price. Accurate estimation of the state-of-charge (SOC) of a battery pack is important in managing the health and safety of battery packs. This paper proposes a compact radial basis function (RBF) neural model to estimate the state-of-charge (SOC) of lithium battery packs. Firstly, a suitable input set strongly correlated with the package SOC is identified from directly measured voltage, current, and temperature signals by a fast recursive algorithm (FRA). Secondly, a RBF neural model for battery pack SOC estimation is constructed using the FRA strategy to prune redundant hidden layer neurons. Then, the particle swarm optimization (PSO) algorithm is used to optimize the kernel parameters. Finally, a conventional RBF neural network model, an improved RBF neural model using the two stage method, and a least squares support vector machine (LSSVM) model are also used to estimate the battery SOC as a comparative study. Simulation results show that generalization error of SOC estimation using the novel RBF neural network model is less than half of that using other methods. Furthermore, the model training time is much less than the LSSVM method and the improved RBF neural model using the two-stage method.
\end{abstract}

\section{Introduction}

Lithium-ion batteries have been widely used as energy storage devices and in electric vehicles due to their desirable balance of both energy and power densities. Compared with single lithium battery cells, a lithium battery pack with hundreds even thousands of battery cells connected in parallel and series is able to provide the required power in various applications [1-3]. The battery management system (BMS) plays an important role in maintaining safe and efficient operation of the battery. The State-of-Charge (SOC) of li-ion battery pack is a key parameter affecting the battery life, safety and efficient operation $[4,5]$. Based on the accurate estimation of SOC, effective management strategies can be developed to avoid overcharging/overdischarging, prolong the cycle life of batteries, and prevent the occurrence of security incidents [6]. Furthermore, with the correctly estimated SOC information, drivers can also arrange the driving time properly.

Due to the complex nonlinear characteristics of li-ion batteries, SOC cannot be measured directly in real-time applications, and it needs to be inferred using other measurable variables [7]. Since a battery pack may consists of hundreds and even thousands of battery cells, the computation effort for modelling is increased accordingly. Besides, the inconsistency of cells in a battery pack varies along with the life of the battery. Thus, it is a challenge to accurately estimate the SOC of the battery pack. Recently, a number of methods have been proposed to improve the SOC estimation and they can be grouped to three general approaches for the estimation of battery pack SOC. The first approach integrates the cell model into the structure of the battery pack $[8,9]$. However, the inconsistency between different cells in a battery pack is ignored. 
In the second category, the single cell SOC estimation approach is directly extended to battery packs, including open circuit voltage method [10], ampere-hour integral method [11], Kalman filter [12, 13], and the equivalent electric circuit model [8]. These methods treat the battery pack as a "big battery" [14], which makes the SOC estimation simpler and more quick. However, the simple model is based on the precise mechanism of single cells. Due to the inconsistency between different battery cells, estimation error inevitably exists.

The third category includes various statistical methods. Plett first proposed the Bar-Delta Filter method in 2009 [15] which uses a Sigma Point Kalman Filter (SPKF) to estimate the average SOC of the battery pack and Delta Filters to estimate the variance between the cell's characteristics and the average characteristics. However, the accuracy of the battery SOC estimation is a key, which is still a challenge. Dai et al. [16] and Sun and Xiong [14] proposed a dual time-scale Kalman filter, based on the equivalent electrical circuit model (EECM) where the differences in the internal resistance battery cells are considered. The mean SOC model and the differences of battery SOC proposed by Zheng et al. $[17,18]$ use the extended Kalman filter (EKF) based on the cell mean model (CMM) and cell difference model (CDM) to estimate both the mean SOC value of battery cells and their differences, respectively. This method still requires internal information about the battery pack. Deng et al. [19] proposed a data-driven method, and an efficient feature selection method is used to estimate the SOC of a battery pack using an autoregressive Gaussian process regression (GPR) model $[20,21]$. A challenge for the GPR modelling is its computation time $\left(\mathrm{O}\left(\mathrm{N}^{3}\right)\right)$.

In summary, albeit the aforementioned progresses in the battery pack SOC estimation, to develop a simple yet accurate model is still an important issue in real-life battery applications. Data-driven methods [22] have gained a lot of interest in recent years to solve highly nonlinear classification and regression problems. The advantages of datadriven methods are the flexibility and model-free [23] characteristics which make them easy to create new models. As a class of data-driven methods [24], the machine learning approaches, such as support vector regression [25], Kalman filter $[12,13,17]$, and backpropagation (BP) neural networks [26], have been successfully used in SOC estimation and prediction. However, the selection of dataset and input features for building these models is still ad hoc via trial and error.

To overcome some shortcomings in the aforementioned methods for the battery pack SOC estimation, this paper presents an improved RBF method using a fast recursive algorithm (FRA) to estimate the SOC of a battery pack. The FRA method [27] can be used for both neural inputs selection [28] and hidden layer node selection [29-31] in the configuration of RBF networks. Comparing to [32], the average cell temperature, the time mean pack voltage, the time mean pack temperature, and the time mean loop current all over 10 seconds intervals can be also added to the initial candidate pool of input variables, other input candidates can also be included such as the maximum cell voltage, the minimum cell voltage, the average cell voltage, and loop current. The statistical variables are adopted to reduce the complexity of the model and the cell information is used to overcome the inconsistency among single cells. Then, a compact subset of these candidate variables are selected as the model input by the FRA method. On this basis, an improved RBF model built by the FRA method is used to predict the SOC of the battery pack. The proposed RBF model is automatically constructed by the selection of the hidden layer nodes using the FRA method. Furthermore, the parameters of RBF kernel are optimized by particle swarm optimization algorithm (PSO).

The rest of this paper is organized as follows. Section 1 introduces the input selection based on the FRA method. In Section 2, the application of improved RBF neural network for SOC estimation of battery pack is introduced in detail. Furthermore, the experimental and simulation results are compared in Section 3. Finally, Section 4 concludes the paper.

\section{Input Selection Using FRA}

Based on the theory of series expansion, polynomial NARMAX models can achieve the same modelling performance as various neural networks if certain conditions are satisfied [28]. The input selection of RBF neural network is thus simplified to determining the structure of the polynomial NARMAX model. The structure of the polynomial NARMAX model can be efficiently detected by selecting important polynomial terms using the FRA method with low computational complexity [27].

2.1. FRA Method. Consider the following multiple-input single-output system represented by a linear-in-the-parameter model:

$$
y(t)=\sum_{k=1}^{n} \theta_{k} \varphi_{k}(\vec{X}(t))+\varepsilon(t),
$$

where $y(t), \vec{X}(t) \in R^{m}$, and $\varepsilon(t)$ are output variable, input variable vector, and model error at time instant $t$, respectively. Herein, $m$ and $n$ denote the number of input variables and model terms (mapping functions), respectively. $\varphi_{k}$ is the nonlinear mapping function. $\theta_{k}$ are the linear coefficients for the mapping functions.

For given $N$ training samples, the system model is expressed in the following matrix form:

$$
\vec{y}=\boldsymbol{\Phi}(\vec{X}(t)) \Theta+\mathbf{e},
$$

where $\Phi(\vec{X})=\left[\phi_{1}(\vec{X}), \ldots, \phi_{n}(\vec{X})\right]^{T} \in \Re^{N \times n}$ is formed by mapping functions. $\phi_{i}=\left[\varphi_{i}(\vec{X}(1)), \ldots, \varphi_{i}(\vec{X}\right.$ $(N))]^{T}, i=1, \ldots, n . \vec{y}=[y(1), \ldots, y(N)]^{T} \in \mathfrak{R}^{N}$ is the output vector. $\Theta=\left[\theta_{1}, \ldots, \theta_{n}\right]^{T} \in \mathfrak{R}^{n}$ denotes the linear parameters.

Refer to [27], and the minimal cost function using the least square method is given as

$$
\mathbf{E}=\vec{y}^{T} \vec{y}-\widehat{\boldsymbol{\Theta}}^{T} \boldsymbol{\Phi}^{T} \vec{y}
$$

where 
Require the maximum voltage vector $\vec{v}_{\max } \in \mathfrak{R}^{N \times 1}$, the minimum voltage $\vec{v}_{\text {min }} \in \mathfrak{R}^{N \times 1}$, the average voltage $\vec{v}_{\text {avg }} \in \mathfrak{R}^{N \times 1}$, the average temperature $\overrightarrow{\mathrm{tmp}} \in \mathfrak{R}^{N \times 1}$ of the battery cells, the circuit current $\vec{I} \in \Re^{N \times 1}$, the maximal order of time lags for inputs $l_{x}=10$, the maximal order of time lags for output $l_{y}=3$, the maximal number of selected terms $m$, and the minimal training error $e$.

Ensure the SOC vector of the battery pack $\vec{y} \in \mathfrak{R}^{N \times 1}$.

(1) Initialization: form the regression matrix for polynomial term selection.

(2) for $i=1$ to $n$ do

(3) calculate the recursive matrix $\mathbf{A}, A_{y}, \quad a_{j, j}$ and $a y_{j} \quad(j=1, \ldots, m)$ is recursively calculated by $a_{i, j}=0, j<i$

$$
\begin{aligned}
& a_{i, j}={ }^{\phi_{i}^{(i-1)} T} \phi_{i}^{(i-1)}={ }^{\phi_{i} T} \phi_{i}-\sum_{h=1}^{i-1} a_{h, i}^{2} / a_{h, h}, j=i \quad a y_{i}=\phi_{i}^{T} \mathbf{R}_{i-1} \mathbf{y}=\phi_{i}^{T} \mathbf{y}-\sum_{h=1}^{i-1}\left(\left(a_{h, i} a_{h, j}\right) / a_{h, h}\right), 1 \leq i<k \\
& a_{i, j}=\left(\phi_{i}^{(i-1)}\right)^{T} \phi_{j}^{(i-1)}=\left(\phi_{i}\right)^{T} \phi_{j}-\sum_{h=1}^{i-1}\left(\left(a_{h, i} a_{h, j}\right) / a_{h, h}\right), i<j<n
\end{aligned}
$$

(4) calculate the net contribution of the terms using equation (10).

(5) select the significant term.

(6) end for

(7) Input selection: find the order of the time lags from the selected model terms.

Algorithm 1: Input selection using FRA algorithm.

$$
\widehat{\boldsymbol{\Theta}}=\operatorname{argmin} \Theta\|\vec{y}-\Phi \Theta\|_{2}=\left(\boldsymbol{\Phi}^{T} \boldsymbol{\Phi}\right)^{-1} \boldsymbol{\Phi}_{T} \vec{y}
$$

Thus, the minimal cost function is reformulated as follows:

$$
\mathbf{E}=\vec{y}^{T} \vec{y}-\vec{y}^{T} \boldsymbol{\Phi}\left(\boldsymbol{\Phi}^{T} \boldsymbol{\Phi}\right)^{-1} \boldsymbol{\Phi}^{T} \vec{y} .
$$

Use the definitions below:

$$
\left\{\begin{aligned}
\mathbf{M}_{k} & \triangleq \boldsymbol{\Phi}_{k}^{T} \boldsymbol{\Phi}_{k}, \\
\mathbf{R}_{k} & \triangleq I-\boldsymbol{\Phi}_{k}\left(\boldsymbol{\Phi}_{k}^{T} \boldsymbol{\Phi}_{k}\right)^{-1} \boldsymbol{\Phi}_{k}^{T}, \\
\mathbf{R}_{0} & =\mathbf{I}_{N \times N}, \\
k & =0,1, \ldots, n .
\end{aligned}\right.
$$

The variance of the minimal cost function $\mathbf{E}$ induced by an additional mapping function $\phi_{k+1}$ is given as follows:

$$
\Delta \mathbf{E}_{k+1}=\mathbf{E}_{k+1}-\mathbf{E}_{k}=\vec{y}^{T} \mathbf{R}_{k+1} \vec{y}-\vec{y}^{T} \mathbf{R}_{k} \vec{y}
$$

Using the propositions detailed in [27], equation (7) is rewritten as follows:

$$
\Delta \mathbf{E}_{k+1}=\frac{-\left(\vec{y}^{T} \phi_{k+1}^{(k)}\right)^{2}}{\left(\phi_{k+1}^{(k) T} \phi_{k+1}^{(k)}\right)}, \quad k=0,1, \ldots, n-1,
$$

where $\phi_{k+1}^{(k)}=\mathbf{R}_{k} \phi_{k+1}$.

Obviously, the variance $\Delta \mathbf{E}_{k+1}$ only concerns the additional mapping function $\phi_{k+1}$. Then, define the recursive matrix $\mathbf{A}=\left[a_{i, j}\right]_{k \times n}$ and recursive vector $A_{y}=\left[a_{i, j}\right]_{n \times 1}^{T}$, the elements of which are defined as follows:

$$
\begin{aligned}
& a_{k, i} \triangleq\left(\phi_{k}^{(k-1)}\right)^{T} \phi_{i}^{(k-1)}, \quad \text { where } a_{1, i} \triangleq\left(\phi_{1}\right)^{T} \phi_{i} k=1,2, \ldots, n, i=k, \ldots, n, \\
& a_{k, y} \triangleq\left(\phi_{k}^{(k-1)}\right)^{T} \vec{y}, \quad \text { where } a_{1, y} \triangleq\left(\phi_{1}\right)^{T} \vec{y} k=1,2, \ldots, n .
\end{aligned}
$$

Therefore, the net contribution induced by the $\phi_{k+1}$ is expressed as

$$
\Delta \mathbf{E}_{k+1}\left(\phi_{k+1}\right)=\frac{\left(\vec{y}^{T} \phi_{k+1}-\sum_{j=1}^{k}\left(a_{j, y} a_{j, k+1} / a_{j, j}\right)\right)^{2}}{\left(\phi_{k+1}\right)^{T} \phi_{k+1}-\sum_{j=1}^{k}\left(a_{j, k+1}^{2} / a_{j, j}\right)}, \quad k=1,2, \ldots, n .
$$

And the linear coefficients are estimated by

$$
\widehat{\theta}_{j}=\frac{\left(a_{j, y}-\sum_{i=j+1}^{k} \hat{\theta}_{i} a_{j, i}\right)}{a_{j, j}}, \quad j=k, k-1, \ldots, 1 .
$$


Require: selected input variable matrix $\Phi \in \mathfrak{R}_{N \lambda m}$ in equation (2), the variable upper/lower bounds $\left[X_{\min }, X_{\max }\right.$ ] and the velocity upper/lower bounds $\left[v_{\min }, v_{\max }\right]$, the size of the population $l$, the maximum number of iterations $T$, the crossover factors $C R=\left[c_{1}, c_{2}\right] \in[0,1]$, and the acceleration of the particle velocity $w_{i}$.

Ensure: the SOC vector of the battery pack $\vec{y} \in \mathfrak{R}^{N \times 1}$.

(1) Initialization: $\mathbf{R}_{0}=\mathbf{I}_{N \times N}, \mathbf{J}_{0}=1 / N \sum_{t=1}^{N} y(t)^{2} k=0$.

(2) While $\mathbf{J}_{k}>\mathbf{J}_{k+1}$ do

(3)Initialization: Set the initial centers $x_{j, 0}^{1}$ and widths $x_{j, 0}^{2}$ of the RBF basis function, where $j=1, \ldots, l$, thus the initial nonlinear parameters are $\vec{X}_{0,0}=\left[x_{1,0}^{1}, x_{1,0}^{2}\right] ;\left[x_{2,0}^{1}, x_{2,0}^{2}\right], \ldots,\left[x_{l, 0}^{1}, x_{l, 0}^{2}\right]^{T}$. Set the initial velocity $\vec{V}_{0,0}=\left[v_{1,0}^{1}, v_{1,0}^{2}\right] ;\left[v_{2,0}^{1}, v_{2,0}^{2}\right], \ldots,\left[v_{l, 0}^{1}, v_{l, 0}^{2}\right]^{T}$.

(4)for $i=1$ to $T$ do

(5) construct the candidate $\mathrm{RBF}$ basis vectors.

(6) calculate the matrix $\mathbf{R}_{k}$ using $\mathbf{R}_{k+1}=\mathbf{R}_{k}-\left(\mathbf{R}_{k} \psi_{k+1} \psi_{k+1}^{T} \mathbf{R}_{k}\right) /\left(\psi_{k+1}^{T} \mathbf{R}_{k} \psi_{k+1}\right)$ and the recursive matrix A, $A_{y}$ using Algorithm 1, respectively.

(7) calculate the vector $\vec{e}_{k+1}$ using $\vec{e}_{k+1}=\mathbf{R}_{k+1} \vec{y}$.

(8) Find the candidate regressor that gives the minimal PRESS error, and record the minimal PRESS error (index $s$, minimal PRESS error $\mathbf{J}_{i, k}$ ) and the best position of each particle $\vec{p}_{b, i}=\left[x_{s, i}^{1}, x_{s, i}^{2}\right]^{T}$.

(9) compare to the last best position using $\mathbf{J}_{i-1, k}$, obtain the global optimal position $\vec{p}_{g, i}$

(10) update velocity and position using $\vec{V}_{k, i}=w_{i} \vec{V}_{k, i-1}+c_{1} r_{1}\left(p_{b}-X_{i, k}\right)+c_{2} r_{2}\left(\vec{p}_{g, i}-X_{i, k}\right), X_{k, i+1}=X_{k, i}+v$ where $\vec{V}_{k, i}$ and $X_{k, i+1}$ denote the velocity and particle at $i^{\text {th }}$ iteration for $k^{t h}$ selection, $r_{1}$ and $r_{2}$ is the random numbers.

(11)end for

(12)add the candidate feature with the minimal PRESS error to the regression matrix $\Phi, k=k+1$.

(13)end while

(14)Identification: calculate the linear coefficients using equation (11).

Algorithm 2: Compact RBF modelling algorithm using the PSO method.

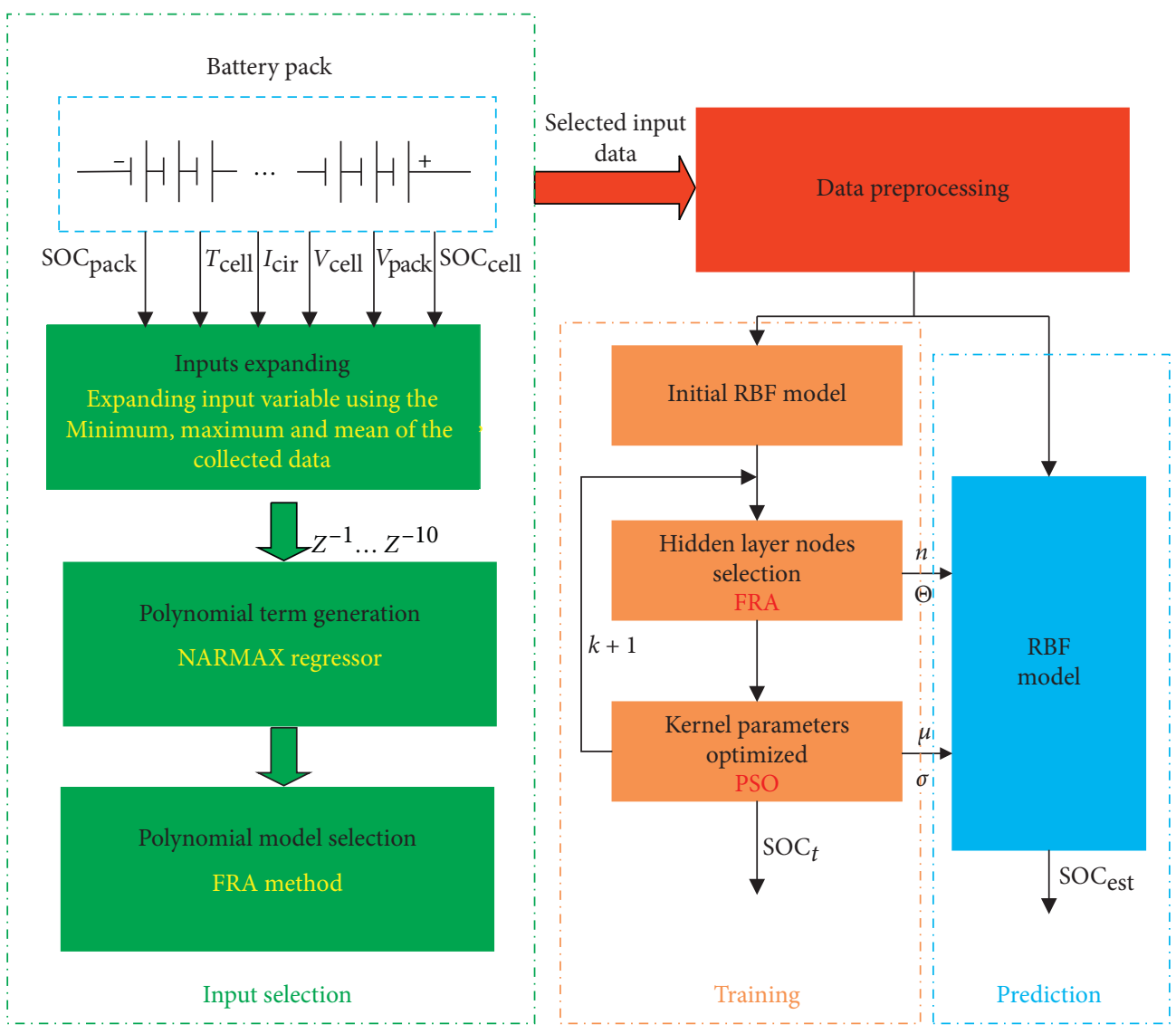

Figure 1: The flowchart of the battery pack SOC estimation. 
TABLE 1: Performance for different input variables using proposed RBF.

\begin{tabular}{|c|c|c|c|c|c|}
\hline Inputs & RMSE (training) & RMSE (validation) & MAX (training) (\%) & MAX (validation) & Time \\
\hline Selected by FRA & 0.041 & 0.087 & 0.6783 & 1.9606 & 114 \\
\hline By experience & 0.042 & 0.9811 & 0.6287 & 2.5228 & 117 \\
\hline
\end{tabular}
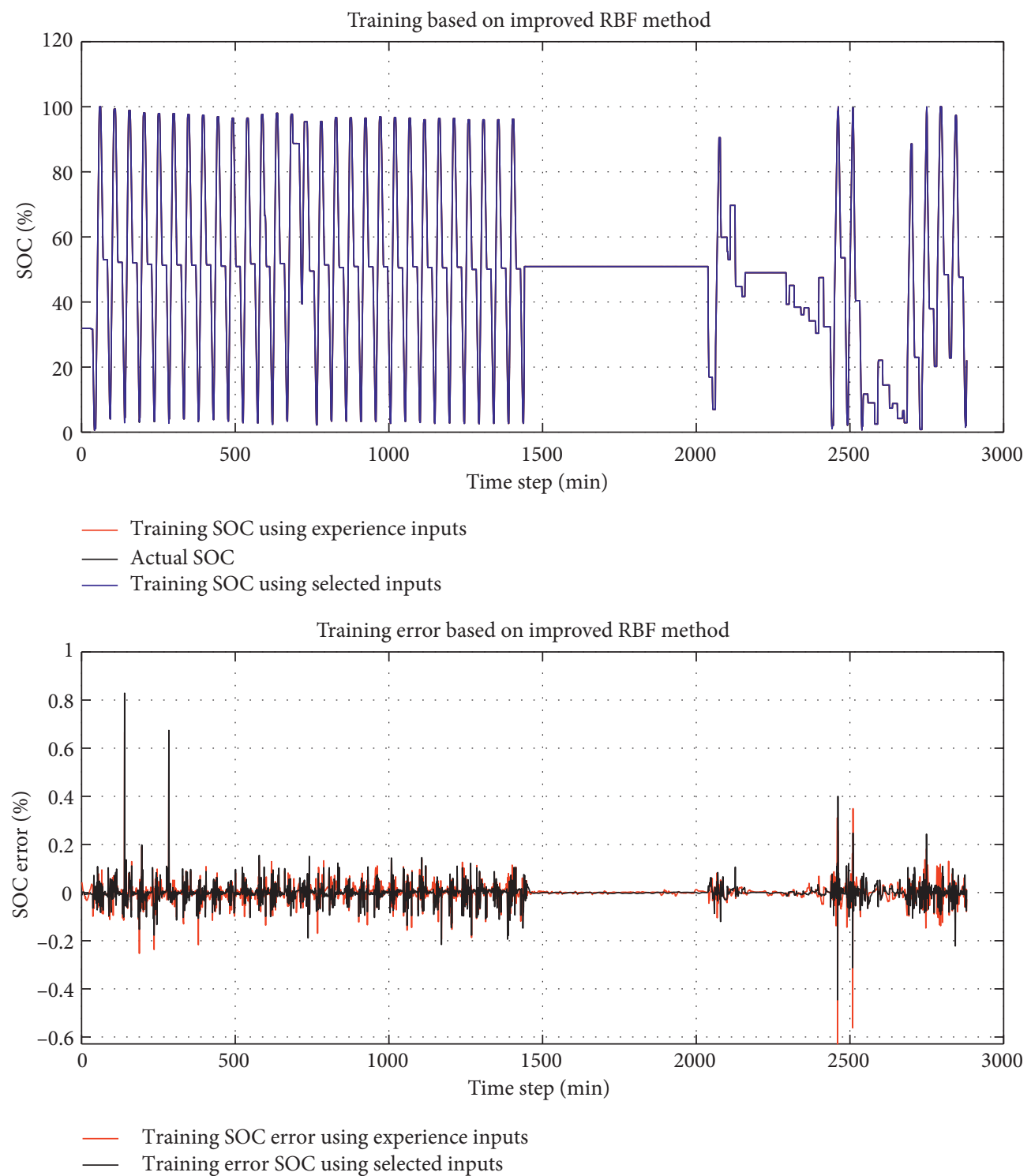

FIgURE 2: Training results for different model inputs using proposed RBF.

2.2. Input Selection for Battery Pack SOC Estimation. For the battery pack SOC estimation, the measured variables including the maximum and minimum voltages of the battery cells, the overall voltage of the battery pack, the circuit current, and the average temperature of the battery cells are all possible candidates as the model inputs and the targeted SOC is considered as model output, respectively. Based on the moving average method, the mean of a time sequence values in the shifting time window can be used to eliminate the random and other noises. Then, the average of the pack voltage, the pack temperature, and loop current for every 10 seconds interval is calculated as the time mean pack voltage, the time mean pack temperature, and the time mean loop current, respectively. Both the measured variables and the statistical variables are considered as augmented variables for estimating the battery pack SOC. The SOC of a battery pack is a time sequence, so both the model dependent variables and the model output measured in the past are critical to the estimation of next SOC value. However, not all of the historical data are needed for SOC estimation, so the maximum order of time lags for these input variables should be determined in advance.

To select the RBF neural network inputs, the problem is converted into the polynomial model construction. Thus, the input selection problem is formulated as equation (1).

Herein, the mapping functions are selected using the following polynomial terms: 

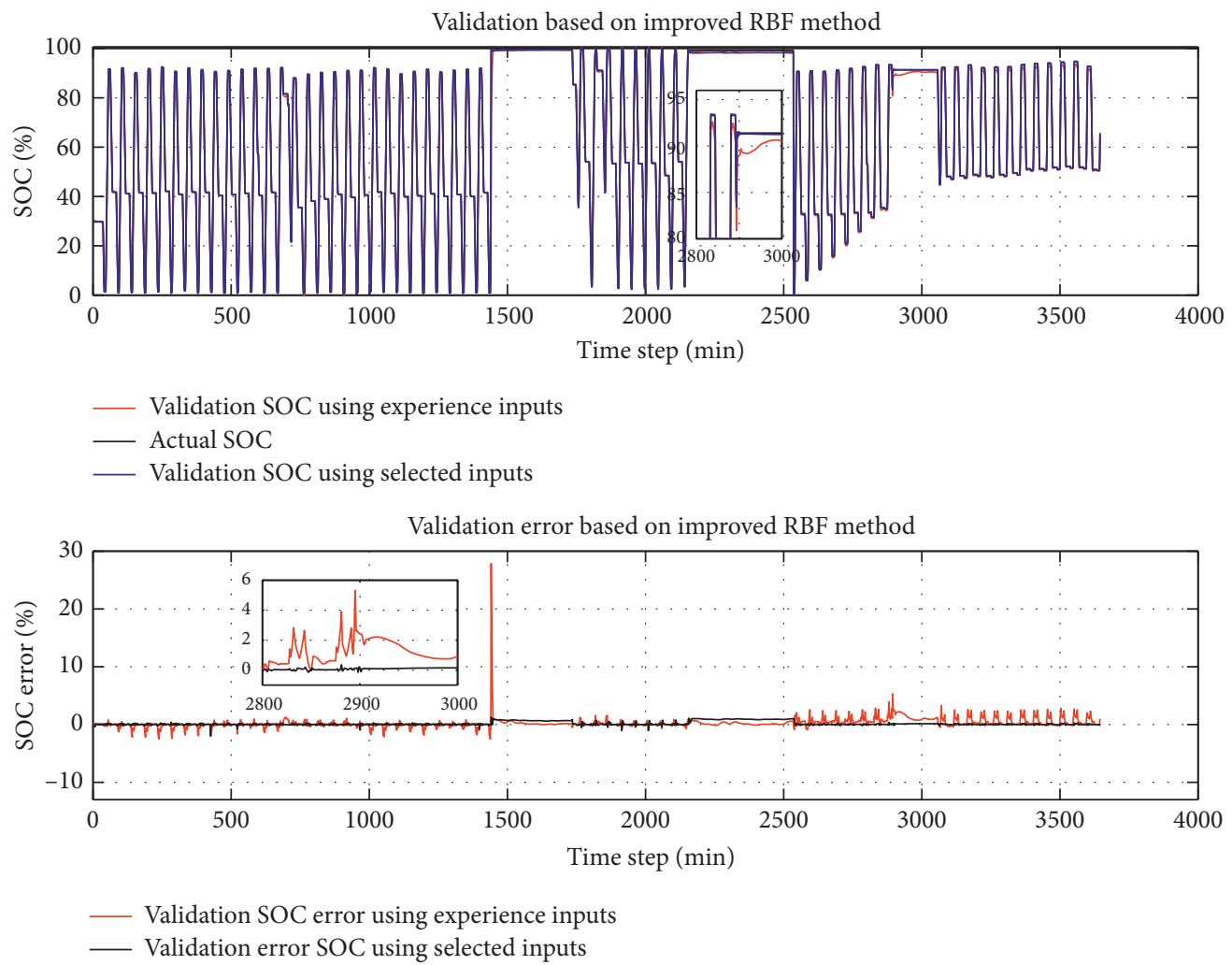

FIGURE 3: Validation results for different model inputs using proposed RBF.

TABle 2: Performance for the SOC estimation using different methods.

\begin{tabular}{lccccc}
\hline Method & RMSE (training) (\%) & RMSE (validation) (\%) & MAX (training) (\%) & MAX (validation) (\%) & Time (s) \\
\hline Proposed RBF & 0.041 & 0.087 & 0.4458 & 2.0232 \\
General RBF & 0.041 & 0.1539 & 0.6956 & 2.0904 \\
LSSVM & 0.042 & 0.1179 & 0.8289 & 1.8128 \\
TSS_RBF & 0.044 & 0.1136 & 0.7893 & 1.9603 \\
\hline
\end{tabular}

$$
\begin{aligned}
\phi_{k}=\prod_{j=n_{y 1}}^{n_{y i}} y(t-j) \prod_{k=1}^{m} \prod_{j=n_{x k 1}}^{n_{x k i}} x_{k}\left(t-d_{k}-j\right), & \\
& i=1, \ldots, p, \phi_{0}=1,
\end{aligned}
$$

where $0 \leqq n_{y k 1} \leqq \cdots \leqq n_{y k i} \leqq l_{y}, 0 \leqq n_{x k i} \leqq \cdots \leqq n_{x k i} \leqq l_{x}$, and $l_{x}=10$ and $l_{y}=3$.

Then, the neural network model inputs can be identified by selecting the most significant polynomial terms using the FRA method. The following input selection method is detailed in Algorithm 1.

\section{Improved RBF Model for the SOC Estimation}

This paper aims to develop an accurate yet simple model for battery pack SOC estimation. Deng et al. proposed a two stage algorithm based on the leave-one-out method [30] to increase the performance of RBF neural networks. The selection procedure is automatically terminated by predicted-residual-sums-of-squares (PRESS) error so that the constructed RBF neural model is parsimonious and accurate. In this paper, the FRA method is used instead of the two stage algorithm for RBF neural network construction, which reduces the modelling complexity. In order to ensure the accuracy of the model, particle swarm optimization (PSO) algorithm is used to optimize the kernel parameters.

3.1. General RBF Neural Network. A RBF neural model can be formulated as a linear-in-the-parameters model like equation (1) as follows:

$$
y(t)=\sum_{k=1}^{n} \theta_{k} \varphi_{k}\left(\vec{X}(t) ; c_{k} ; \sigma_{k}\right)+\varepsilon(t)
$$

where the additional parameters $\varphi_{k}\left(\vec{X}(t) ; c_{k} ; \sigma_{k}\right)$ is the radial basis activation function for the hidden nodes which is often chosen as a Gaussian function. $c_{k} \in R^{m}$ is the centers, and $\sigma_{k} \in R^{1}$ denotes the RBF widths.

Similar to equation (2), the RBF neural model is formulated in the matrix form as follows: 

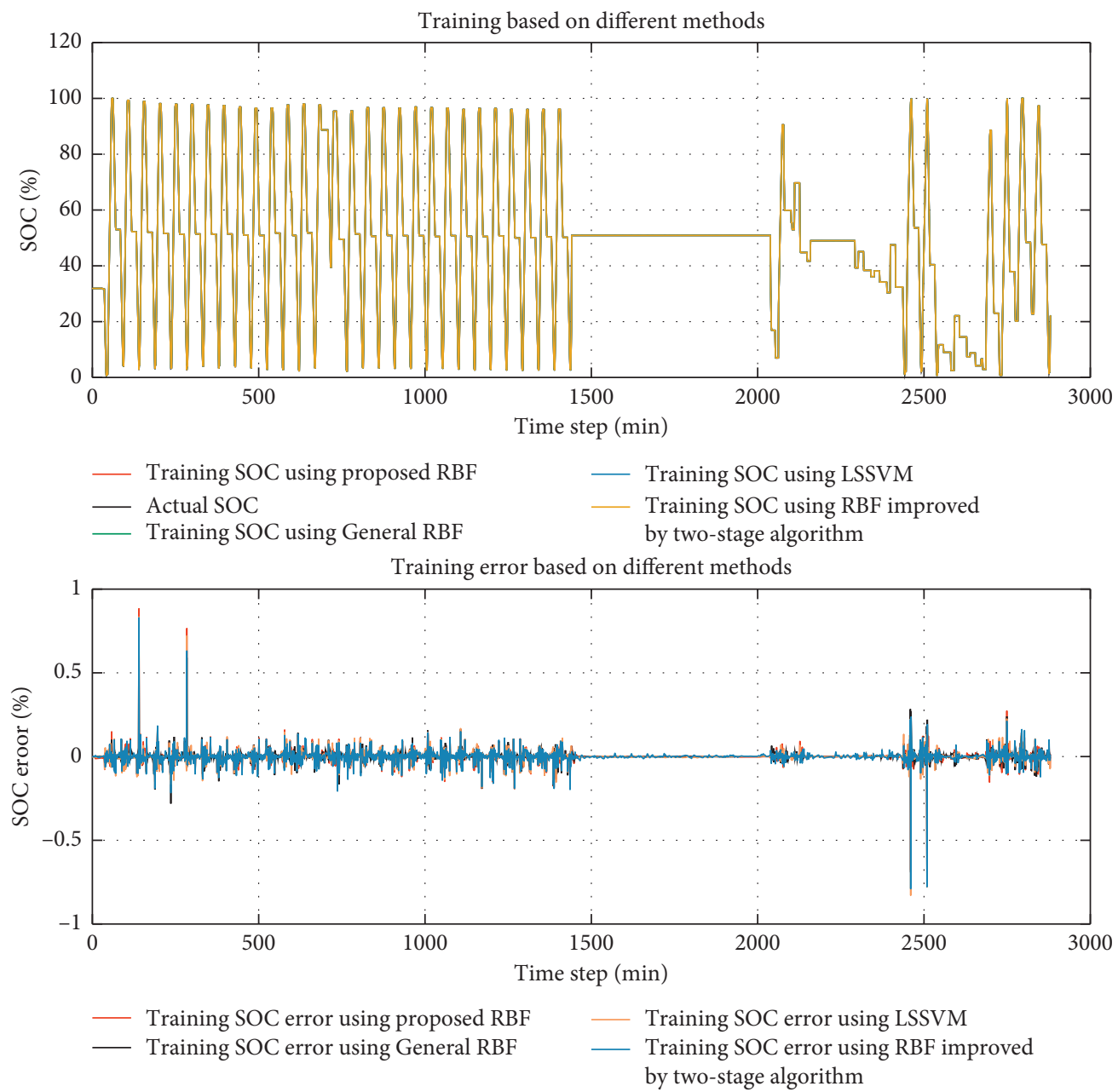

FIgURE 4: Training results of the FUDS process using different weights.

$$
\vec{y}=\boldsymbol{\Phi}(\mathbf{X}, \vec{c}, \vec{\sigma}) \Theta+\vec{e}
$$

where $\Phi=\left[\phi_{1}, \ldots, \phi_{n}\right]^{T} \in R^{N \times n}$ is the output matrix of the hidden nodes. $\phi_{i}=\left[\varphi_{i}(\vec{X}(1), \vec{c}, \vec{\sigma}), \ldots, \quad \varphi_{i}(\vec{X}\right.$ $(N), \vec{c}, \vec{\sigma})]^{T}, i=1, \ldots, n$

3.2. Improved RBF Neural Model. The performance of the RBF neural model is related to the number of the hidden layer nodes and the kernel parameters. Therefore, the construction of RBF network can be regarded as an optimization problem which depends on the number of hidden layer nodes, kernel parameters, and connection weights. In order to improve the accuracy and real-time performance of Li-ion battery pack SOC estimation, the FRA method is used to establish an accurate and compact RBF neural model.

Using the improved RBF neural model based on the FRA method, the hidden layer nodes are selected according to the net contribution of the hidden layer node output. At the same time, the nonlinear kernel parameters are optimized by the particle swarm optimization method. Particle swarm optimization (PSO) [33] is a nonlinear parameter optimization algorithm based on swarm intelligence, and it has been widely used for nonlinear parameter optimization. The method is simple and easy to implement, it is applied to the parameter optimization of RBF kernel function. According to [30], leave-one-out (LOO) crossvalidation and associated predicted-residualsums-of-squares (PRESS) error are used as an index to select hidden layer nodes and automatically break the selection procedure. The hidden layer nodes are selected with the maximal reduced PRESS error. Thus, the net contribution is changed to the following equation:

$$
\mathbf{J}_{k}=\frac{1}{N} \sum_{t=1}^{N} \frac{e_{k}^{2}(t)}{\mathbf{R}_{k}^{2}(t, t)}, \quad k=1,2, \ldots, n,
$$

where $N$ and $n$ is the number of the samples and the max number of the hidden layer nodes, respectively. $e_{k}(t)$ and $\mathbf{R}_{k}(t, t)$ is the model error and the defined matrix $\mathbf{R}_{k}$ in equation (6) at time instant $t$, respectively.

Based on this net contribution, the improved RBF neural networks optimized by the PSO method is shown in Algorithm 2. 

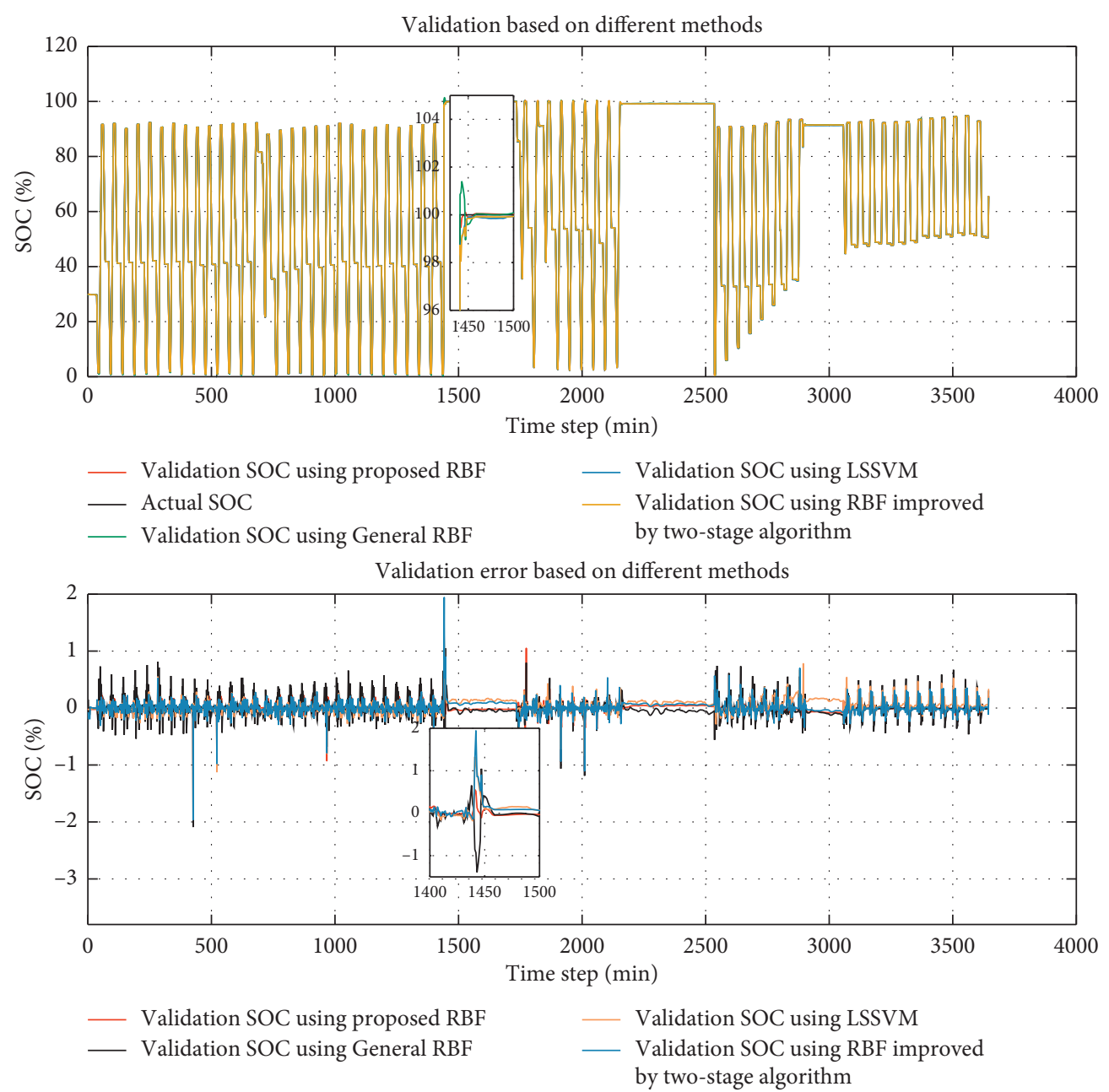

FIGURE 5: Validation results of the FUDS process using different weights.

3.3. Battery Pack SOC Estimation. As mentioned earlier, the battery pack SOC is estimated using the improved RBF neutral network. The schematic diagram of the proposed method for the battery pack SOC estimation is illustrated in Figure 1.

From Figure 1, there are three parts in the proposed method. In the first part, the inputs are determined from the measurements including the voltage of the battery cell $\left(V_{\text {cell }}\right)$, the voltage of the battery pack $\left(V_{\text {pack }}\right)$, the terminal current $\left(I_{\text {cir }}\right)$, the temperature of the battery cell $\left(T_{\text {cell }}\right)$, the SOC of the battery cell $\left(\mathrm{SOC}_{\text {cell }}\right)$, and the SOC of the battery pack $\left(\mathrm{SOC}_{\text {pack }}\right)$. Before the model inputs are determined by the FRA method, the candidate inputs are expanded by finding the maximum, the minimum, and the mean of $V_{\text {pack }}, T_{\text {cell }}$, and $I_{\text {cir }}$. Then, the delayed sequence obtained by using delay operator $\left(z^{-1}, \ldots, z^{-10}\right)$ is adopted to produce the polynomial terms. Thus, the inputs are selected from the terms in the resultant nonlinear autoregressive moving average with exogenous inputs (NARMAX) model. In the second part, the improved RBF model is trained using the FRA method combined with the PSO method. Finally, the
SOC of the battery pack is predicted using the built RBF model in which the kernel parameters $(\mu, \sigma)$, the number of the hidden layer $(n)$ nodes, and the weights to the outputs $(\Theta)$ are optimized by PSO [33].

\section{Simulation Results}

We first consider a package with 216 battery cells of 18650 types connected in series. 8 battery packs in the same configuration were tested. In these tests, the circuit current, the terminal voltage of the battery pack, the terminal voltages of each cell individual, and the temperature between two battery cells are measured every $1 \mathrm{~s}$. The SOC of the battery pack and the battery individual cell are all estimated every 1 s by the battery management system.

The data collected from a battery pack are often too large to be used to establish the estimation model. The ageing of battery capacity can be ignored in a short period, the training samples are selected every $30 \mathrm{~s}$ to build the improved RBF model. Then, the model inputs are chosen by FRA for the battery pack SOC estimation. 
Using the FRA method, the maximum voltage $v_{-} \max (t)$ of the battery cells, the minimum voltage $v_{-} \min (t)$ of the battery cells, the average voltage of the battery pack $v_{-}$avg $(t)$, the voltage of the battery pack $v(t)$, the mean voltage $v_{-} m(t)$ of past 10 measurements, the mean current $i_{-} m(t)$ of past 10 measurements, the mean temperature tmp_m $m(t)$ of past 10 measurements, the circuit current $i(t)$ and the average temperature $\operatorname{tmp}(t)$ of the battery cells, and the estimated SOC $\operatorname{soc}(t)$ are adopted as the inputs and output, respectively. Finally, $v_{-}$avg $(t-4), i(t), i(t-6), i(t-7), i(t-8)$, $v(t-7), \quad v_{-} \max (t-8), \quad v_{-} m(t), v_{-} m(t-8), i_{-} m(t-2)$, tmp_ $m(t-2)$, tmp_ $m(t-8)$, and $\operatorname{soc}(t-1)$ are selected. To verify the selected inputs, the improved RBF model for the SOC estimation is built using the selected inputs compared to the inputs selected by experience (trial and error). The performance using different inputs are shown in Table 1.

In Table 1, the RMSE (root mean square error) and the max absolute error are shown. Clearly, the model using the selected inputs performs much better, with the RMSE of the absolute error is almost always within \pm 0.08 . The simulations are illustrated in Figure 2 and 3. It is shown that the SOC estimation is more accurate using the selected inputs of which the generalization error is less than that using experience inputs.

Then, the proposed model is compared with the conventional RBF method, the general least square support vector method (LSSVM), and the improved RBF neural model and optimized by the two-stage method (TSS_RBF) [30]. The performance of the three methods is shown in Table 2.

According to Table 2, the proposed RBF method took more time than the conventional RBF method in training the model, but the validation RMSE of the proposed RBF model is just half of that using the general RBF model. While the LSSVM model takes almost 50 times longer to train than the proposed RBF model and the improved RBF neural model by the two stage method takes almost 50 times longer to train than the proposed RBF model. Meanwhile, the validation RMSE of the proposed RBF model is $0.02 \%$ lower than the other methods. The simulation results are shown in Figures 4 and 5. It is clear that the proposed RBF model has excellent generalization capability to obtain more accurate SOC than the other methods.

\section{Conclusions}

In order to estimate the SOC of battery pack accurately, it is necessary to adopt the data-driven method to handle the inconsistencies among the cells in a battery pack. This paper first uses the FRA method to select the input variables to improve the precision of the model because the inputs features are important to ensure the accuracy of the RBF neural networks. The experiment results show that better SOC estimation results can be achieved when a compact set of model inputs is selected. Then, the FRA method is further used to improve construction RBF neural network for battery pack SOC estimation. The hidden nodes of RBF neutral networks are again selected using the FRA method, and the particle swarm optimization algorithm is used to optimize the kernel parameters. The results show that the improved RBF model can achieve high estimation accuracy at acceptable time costs.

\section{Data Availability}

The processed data used to support the findings of this study are included within the article. The data source is provided by the partner of Shenzhen Institute of Advanced Technology, Chinese Academy of Sciences, and can be obtained from the corresponding author upon request.

\section{Conflicts of Interest}

The authors declare that they have no conflicts of interest.

\section{Acknowledgments}

This paper was partially funded by the NSFC under Grant 61673256, Key Project of Science and Technology Commission of Shanghai Municipality under Grant 19500712300, and 111 Project under Grant D18003.

\section{References}

[1] C. Zhang, K. Li, L. Pei, and C. Zhu, "An integrated approach for real-time model-based state-of-charge estimation of lithium-ion batteries," Journal of Power Sources, vol. 283, pp. 24-36, 2015.

[2] F. Feng, X. Hu, L. Hu, F. Hu, Y. Li, and L. Zhang, "Propagation mechanisms and diagnosis of parameter inconsistency within li-ion battery packs," Renewable and Sustainable Energy Reviews, vol. 112, pp. 102-113, 2019.

[3] K. Liu, X. Hu, Z. Yang, Y. Xie, and S. Feng, "Lithium-ion battery charging management considering economic costs of electrical energy loss and battery degradation," Energy Conversion and Management, vol. 195, pp. 167-179, 2019.

[4] Z. Cheng, L. Kang, D. Jing, and S. Song, "Improved real-time state-of-charge estimation of lifepo4 battery based on a novel thermoelectric model," IEEE Transactions on Industrial Electronics, vol. 64, no. 1, p. 199, 2016.

[5] J. Meng, G. Luo, and F. Gao, "Lithium polymer battery stateof-charge estimation based on adaptive unscented kalman filter and support vector machine," IEEE Transactions on Power Electronics, vol. 31, no. 3, pp. 2226-2238, 2016.

[6] K. S. R. Mawonou, A. Eddahech, D. Dumur, E. Godoy, D. Beauvois, and M. Mensler, "Li-ion battery pack soc estimation for electric vehicles, IECON," in Proceedings of the 44th Annual Conference of the IEEE Industrial Electronics Society, pp. 4968-4973, Washington, DC, USA, October 2018.

[7] Y. He, X. Liu, C. Zhang, and Z. Chen, "A new model for stateof-charge (soc) estimation for high-power li-ion batteries," Applied Energy, vol. 101, p. 808, 2013.

[8] J. Li and M. S. Mazzola, "Accurate battery pack modeling for automotive applications," Journal of Power Sources, vol. 237, pp. 215-228, 2013.

[9] C. Sen and N. C. Kar, "Battery pack modeling for the analysis of battery management system of a hybrid electric vehicle," in Proceedings of the Vehicle Power and Propulsion Conference, pp. 207-212, IEEE, Brussels, Belgium, December 2009.

[10] S.-L. Wang, C. Fernandez, C.-Y. Zou et al., "Open circuit voltage and state of charge relationship functional optimization for the working state monitoring of the aerial lithium- 
ion battery pack," Journal of Cleaner Production, vol. 198, pp. 1090-1104, 2018.

[11] M.-H. Chang, H.-P. Huang, and S.-W. Chang, "A new state of charge estimation method for lifepo4 battery packs used in robots," Energies, vol. 6, no. 4, pp. 2007-2030, 2013.

[12] G. L. Plett, "Extended kalman filtering for battery management systems of LiPB-based HEV battery packs," Journal of Power Sources, vol. 134, no. 2, pp. 262-276, 2004.

[13] S. Sepasi, R. Ghorbani, and B. Y. Liaw, "Improved extended kalman filter for state of charge estimation of battery pack," Journal of Power Sources, vol. 255, pp. 368-376, 2014.

[14] F. Sun and R. Xiong, "A novel dual-scale cell state-of-charge estimation approach for series-connected battery pack used in electric vehicles," Journal of Power Sources, vol. 274, pp. 582-594, 2015.

[15] G. L. Plett, "Efficient battery pack state estimation using bardelta filtering, EVS24 international battery," in Proceedings of the Hybrid and Fuel Cell Electric Vehicle Symposium, Stavanger, Norway, May 2009.

[16] H. Dai, X. Wei, Z. Sun, J. Wang, and W. Gu, "Online cell soc estimation of li-ion battery packs using a dual time-scale kalman filtering for ev applications," Applied Energy, vol. 95, pp. 227-237, 2012.

[17] Y. Zheng, W. Gao, M. Ouyang, L. Lu, L. Zhou, and X. Han, "State-of-charge inconsistency estimation of lithium-ion battery pack using mean-difference model and extended kalman filter," Journal of Power Sources, vol. 383, pp. 50-58, 2018.

[18] Y. Zheng, M. Ouyang, L. Lu et al., "Cell state-of-charge inconsistency estimation for lifepo4 battery pack in hybrid electric vehicles using mean-difference model," Applied Energy, vol. 111, pp. 571-580, 2013.

[19] Z. Deng, X. Hu, X. Lin, Y. Che, L. Xu, and W. Guo, "Datadriven state of charge estimation for lithium-ion battery packs based on gaussian process regression," Energy, vol. 205, Article ID 118000, 2020.

[20] K. Liu, Y. Li, X. Hu, M. Lucu, and W. D. Widanage, "Gaussian process regression with automatic relevance determination kernel for calendar aging prediction of lithium-ion batteries," IEEE Transactions on Industrial Informatics, vol. 16, no. 6, pp. 3767-3777, 2020.

[21] K. Liu, X. Hu, Z. Wei, Y. Li, and Y. Jiang, "Modified gaussian process regression models for cyclic capacity prediction of lithium-ion batteries," IEEE Transactions on Transportation Electrification, vol. 5, no. 4, pp. 1225-1236, 2019.

[22] Y. Guo, Z. Yang, S. Feng, and J. Hu, "Complex power system status monitoring and evaluation using big data platform and machine learning algorithms: a review and a case study," Complexity, vol. 2018, pp. 1-21, 2018.

[23] Y. Li, K. Liu, A. M. Foley et al., "Data-driven health estimation and lifetime prediction of lithium-ion batteries: a review," Renewable and Sustainable Energy Reviews, vol. 113, Article ID 109254, 2019.

[24] T. Peters, "Data-driven science and engineering: machine learning, dynamical systems, and control," Contemporary Physics, vol. 60, no. 4, p. 1, 2019.

[25] E. Ipek, M. K. Eren, and M. Yilmaz, "State-of-charge estimation of li-ion battery cell using support vector regression and gradient boosting techniques," in Proceedings of the 2019 the International Aegean Conference on Electrical Machines and Power Electronics (ACEMP) 2019 International Conference on Optimization of Electrical and Electronic Equipment (OPTIM), pp. 604-609, Istanbul, Turkey, August 2019.
[26] Y. Guo, Z. Zhao, and L. Huang, "Soc estimation of lithium battery based on improved bp neural network," in Proceedings of the 8th International Conference on Applied Energy, 2016, pp. 11, ICAE, Beijing, China, October 2016.

[27] K. Li, J.-X. Peng, and G. W. Irwin, "A fast nonlinear model identification method," IEEE Transactions on Automatic Control, vol. 50, no. 8, pp. 1211-1216, 2005.

[28] K. Li and J.-X. Peng, "Neural input selection-a fast modelbased approach," Neurocomputing, vol. 70, no. 4-6, pp. 762-769, 2007.

[29] J. Deng, K. Li, E. Harkin-Jones, M. Fei, and S. Li, “A novel two stage algorithm for construction of rbf neural models based on a-optimality criterion," in Proceedings of the Natural Computation (ICNC), 2013 Ninth International Conference on IEEE, IEEE, pp. 7, Shenyang, China, July 2013.

[30] J. Deng, K. Li, and G. W. Irwin, "A two-stage algorithm for automatic construction of rbf neural models," in Proceedings of the MELECON 2010-2010 15th IEEE Mediterranean Electrotechnical Conference, pp. 166-171, IEEE, Valletta, Malta, February 2010.

[31] W. Liu, Z. Yang, and K. Bi, "Forecasting the acquisition of university spin-outs: an rbf neural network approach," Complexity, vol. 2017, Article ID 6920904, 8 pages, 2017.

[32] Z. Chen, B. Xia, and C. C. Mi, "A novel state-of-charge estimation method for lithium-ion battery pack of electric vehicles," in Proceedings of the IEEE Transportation Electrification Conference and Expo (ITEC), Dearborn, MI, USA, June 2015.

[33] R. Li, S. Xu, S. Li et al., "State of charge prediction algorithm of lithium-ion battery based on pso-svr cross validation," IEEE Access, vol. 8, pp. 10234-10242, 2020. 